\title{
Resource Allocation Models for Cognitive Radio Networks: A Study
}

\author{
Vanita Rana \\ E\&EC Department \\ PEC University of Technology \\ Sector 12, Chandigarh, India
}

\author{
Indu Bala \\ E\&EC Department \\ UCOE, Punjabi University \\ Patiala, India
}

\author{
Neelu Jain \\ E\&EC Department \\ PEC University of Technology \\ Sector 12, Chandigarh, India
}

\begin{abstract}
In this paper, resource allocation models for cognitive radio networks (CRN) using game theory are presented. The study includes the concept of cognitive radio (CR) networks, cooperative and non-cooperative game theory, and modeling of strategic interaction process for CR enabled secondary users. The prime objective of the present study is to compare existing game models for CR networks in terms of concept, approach, system model, and issues associated with each model. At last, conclusion for implementing game models in the cognitive radio networks for effective management of available radio resources is given to provide direction to researchers for future work.
\end{abstract}

\section{Keywords}

Cognitive Radio (CR), Cognitive radio network (CRN), Secondary Users (SUs), Primary users (PUs), Quality of Service (QoS), Wireless service provider(WSP), Game Theory, Nash Equilibrium.

\section{INTRODUCTION}

One of the major challenges in practical implementation of current and future wireless systems is opportunistic spectrum sharing. A CR system provides solution by allowing the secondary users (unlicensed users) to share a part of underutilized or partially utilized spectrum with primary users (legacy users). The term Cognitive Radio was introduced by J. Mitola in 1991 as an intelligent radio communication system which is capable to adapt new transmission parameters in response to the need of user demands and variable environment conditions [1]. Later in 2002, the Federal Communications Commission also suggested that many licensed spectrum bands are under-utilized and some spectrum bands are crowded with over utilization [2]. This fact motivates the development of new technologies and standards in wireless communication systems to use the available spectrum efficiently.

A CR system can be defined as a radio capable of sensing channel conditions and presence of users, learn and predict the efficient way of using the available spectrum and adapting its operation parameters [3]. A CR system can exploit the licensed bands either during the absence of their primary users or by judiciously computing their transmission power in order to benefit from the underutilized portion of the spectrum. With the introduction of CR concept, future wireless systems are expected to have a) much greater spectrum allocations at untapped mm-wave frequency bands, b) highly directional beam-forming antennas at both the mobile device and base station, c) longer battery life, d) lower outage probability, e) much higher bit rates in larger portions of the coverage area, f) lower infrastructure costs, g) and higher aggregate capacity for many simultaneous users in both licensed and unlicensed spectrum [4]. Different functionalities of a cognitive radio system are shown in fig.1.

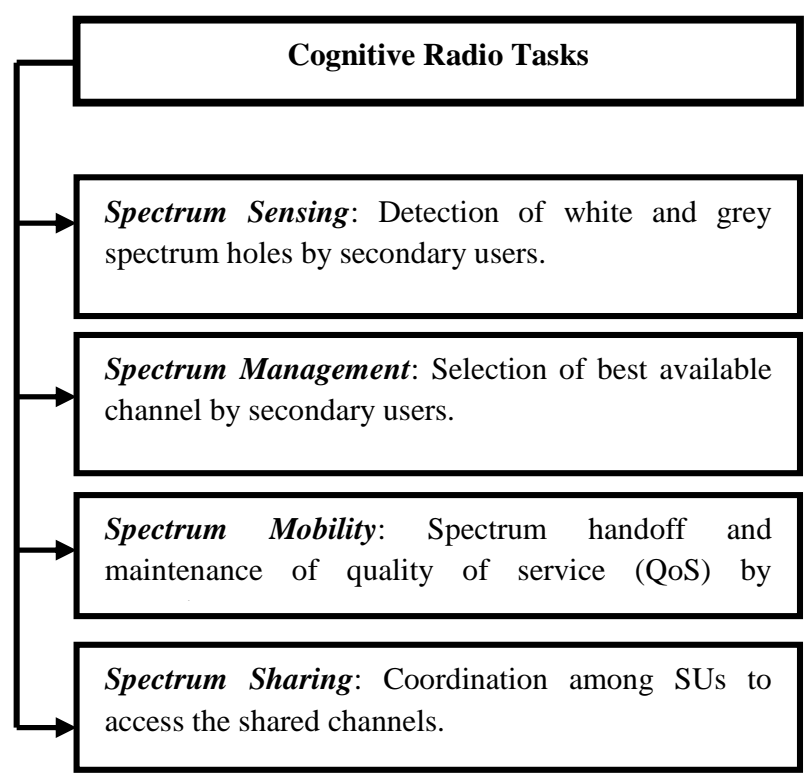

Fig 1 : Functionalities of a CR system.

The present study focuses on the problem of radio resource management using game theory based models. Various approaches used by researchers to model the resource allocation problem have been discussed to provide a comparative evaluation with focus on features, approach and limitations of each model along with possible direction for future work. The paper outline is as follows. The concepts of game theory for CR networks is discussed in section 2 . Section 3 presents game theory based models and comparison of existing techniques for spectrum allocation, finally conclusions are drawn in section 4.

\section{GAME THEORY FOR COGNITIVE RADIO NETWORKS}

J.V. Neumann and O. Morgenstern introduced game theory in 1944[5]. With the help of game theory, it is possible to model the situations where the players/ decision makers have to perform some action. Game Theory is as an important tool used successfully to understand cooperation and conflict between individuals in many fields, such as social sciences, biology, engineering, computer science, etc. It has been already used in the field of communication to understand and find solutions for congestion control, routing, power control, topology control, and trust management. 
Games, according to Game theory, can be cooperative or noncooperative. A non-cooperative game is one in which players make decisions independently. All choices are decided by the players based on their own self-interest, presumably without sharing knowledge. In the strategic form, the payoff for a given player depends on the strategy of that player and all other participating players. The rules and all available strategies are assumed to be common knowledge. There are no unfair advantages or insider knowledge [5]. In contrast to a non-cooperative game, a cooperative game can be used to analyze the optimal strategies for a group of individuals. This includes enforcing collaboration between groups of individuals to jointly improve their position in a game based on theories proposed by J. Nash. This is also known as nash equilibrium which characterizes mutually consistent strategies of the players.

While modeling a CRN using game theory, all secondary users can be considered as players or decision makers, who interact according to a set of rules. In CR networks, the users make intelligent decisions on their spectrum usage and operating parameters. These decisions are based on the sensed spectrum dynamics and actions adopted by other users. Furthermore, users who compete for spectrum resources may have no incentive to cooperate with each other and they may behave selfishly. Resource allocation approach for a cognitive radio environment can be categorized as shown below in fig. 2. Possible criteria for resource allocation and challenges associated with these techniques are also shown in fig. 2 . Some of the basic terms used in game theory are discussed in context to CR systems.

Action: Decision made by secondary users (SUs) on the basis of resource allocation strategy. The single action is denoted by an 'a', while the set of all possible actions is denoted as ' $\mathrm{A}$ '.

Thus, a decision maker is to select a single action $\mathrm{a} \in \mathrm{A}$ from a space of all possible actions.
State Space: The decision process is affected by the unknown quantity $\theta \in \Theta$ which signifies the state of nature. The set of all possible states of nature is denoted by ' $\Theta$ '. Thus, a decision maker perceives that a particular action 'a' results in a corresponding state ' $\theta$ '.

Loss Function: The objectives of a decision maker are described as a real-valued loss function $\mathrm{L}(\theta$, a) which measures the loss (or negative utility) of the consequence $\mathrm{c}(\theta$, a).

Risk Function: For each state ' $\theta$ ', the risk function $\mathrm{R}(\theta, \delta)$ is the expected loss(over $\mathrm{X}$ ) incurred in using $\delta(\mathrm{x})$. It is defined by

$$
R(\theta, \delta)=E_{\theta}^{X}[L(\theta, \delta(X)]
$$

Decision Rule: If a decision maker is to observe an outcome $\mathrm{X}=\mathrm{x}$ and then choose a suitable action $\delta(\mathrm{x}) \in \mathrm{A}$. Aim is to minimize the loss $\mathrm{L}(\theta, \delta(\mathrm{x}))$.

Utility Functions: The quantification of a decision maker's preferences is described by a utility function $u(e, x, a, \theta)$ which is assigned to a particular conduct of experiment ' $e$ ', a resulting observed outcome ' $\mathrm{x}$ ', choosing a particular action 'a', with a corresponding state ' $\theta$ '. It defines value of observed quality of service. QoS maintenance includes both power conservation and SU's wish to have high signal to interference plus noise ratio (SINR) which defines the channel condition.

Strategy: To maximize the payoff each player adopts some strategy. Strategy of a player can comprise of a single move or set of moves. A pure strategy defines an action that a player will follow in every possible attainable situation in a game whereas a mixed strategy gives the probability that action ' $a$ ' will be played.

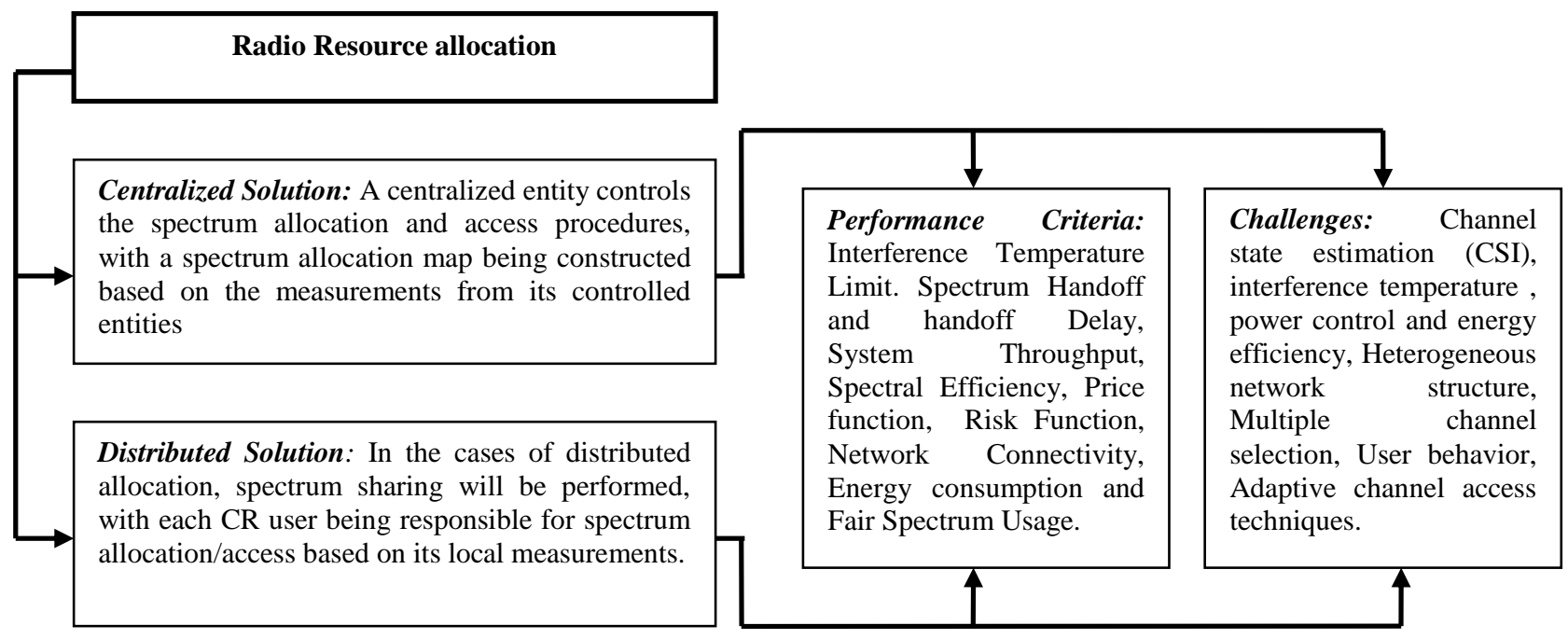

Fig 2. Overview of Radio resource allocation techniques.

State of Equilibrium: It is a state which satisfies most of the players. Under state of equilibrium, all decisions are set and best possible solution for all players is achieved. 


\section{SPECTRUM ALLOCATION MODELS USING GAME THEORY}

A single cell spectrum sharing model with single primary user (PU) and multiple SUs for cognitive radio systems is shown in fig. 3. As discussed in the previous section, it is possible to model CR network systems as a game model. With every model, we can map game players as secondary users who may work in coordination or non coordination to achieve a common goal of spectrum sharing.

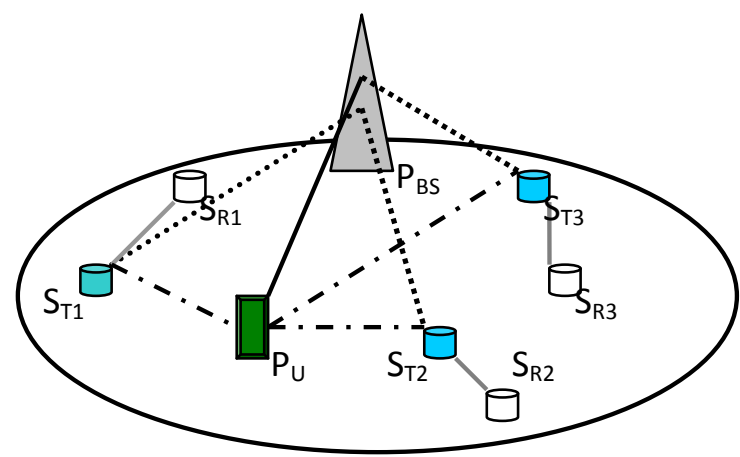

Fig 3 : General layout of a single cell CR network.

\section{Note: PBS: Primary base station, PU: primary user,}

STi:Secondary transmitter, SRi: Secondary receiver.

Performance of any model is influenced by many parameters. Some of these parameters are a) number of licensed and unlicensed users sharing the same cell in CR networks, b) channel sensing technique and its accuracy, c) switching from one channel to another, d) energy cost involved due to spectrum handoff, e) transmission power allocation and $\mathrm{f}$ ) transmission rate. In [6-8], channel model, interference and propagation issues for cognitive radio systems and channel state prediction methods are discussed. the study focuses on shadowing effect and time varying nature of wireless channels. The standardized channel models for both fixed and wireless communication systems are also summarized in [6]. A study on the distribution of the interference generated by a secondary network to a primary network has been presented in [7].

A non-cooperative auction based approach has been discussed in [9]. The study presents a model with distributed control, considering single cell with a PU and multiple SUs. Spectrum allocation is based on auction process but is not applicable to heterogeneous networks. Oligopoly market competition based modeling of CR networks is presented in [10] with a CRN model consisted of single PU and two SUs. This model suffers from lack of choices for SUs and further improvement is possible by considering multiple PUs based cognitive radio network. In [11], focus is on the calculation of nash equilibrium based rate adaptation and power allocation scheme. To achieve this, static and dynamic markovian game model has been proposed. Limitation of this approach is that as the number of users increase, state space also increases. This issue along with need of better pricing mechanism has been highlighted in the same paper as a direction for future work.

With the aim of maximizing channel capacity, a cooperative game model has been proposed in [12]. The CRN model is made up of Multiple PUs, Multiple SUs. Quality of available channel and interference minimization are the constraints used to compute the channel capacity. Channel quality and data rate are two important parameters which ensure quality of service for both primary and secondary users. Keeping this essential requirement in mind, a non-cooperative game model using vickery clarke grove (VCG) mechanism has been introduced in [13] to guarantee a minimum required data rate for both primary and secondary users.

A different approach for spectrum management has been proposed in [14]. In this paper, CRN is modeled by choosing multiple PUs, SUs, service provider, and spectrum regulator. Separate pricing model has been proposed for PS-MSs and CR-MSs. This spectrum management technique simultaneously considers all the participants for fast and efficient spectrum allocation. In [15], an auction mechanism based on contract theory is presented. The study considered primary operator as a monopolist and an algorithm is used to find channel quality and price of spectrum. However, this model is centrally controlled and SUs may face delay if central allocator gets overloaded. A multiple auctioneer based game model is proposed in [16]. In this model, each SU selects one auctioneer for bidding and each primary operator (PO) keeps on raising the trading price. This process repeats until no PO and SU would like to change his decision. The proposed model increases the complexity by allowing multiple auctioneers to participate in the channel allocation process. Also, there are several rounds of bidding due to which secondary users may face delay in getting channel access. But the model also has advantage that it gives more channel options and access of best suited channel to SUs. A flexible channel cooperation design has been shown in [17] for both centralized and decentralized approach. The concept of time division among primary and secondary users has been used and instead of bidding for a single channel, the model allows bidding for multiple channels.

With advancement in the field of wireless communication, heterogeneous network structures also gained popularity as they allow use of different access technologies. An attempt to observe the impact of spectrum heterogeneity in dynamic spectrum access policies has been made in [18]. A non cooperative game model has been used to model the wireless service provider (WSP)'s spectrum pricing policy and to demonstrate the effect of network heterogeneity on the pricing scheme. In [19], a technique called 'TRUST' has been proposed which allows use of any algorithm for resource allocation which allows auctioneer to implement auctions based on performance requirement. Instead of directly involvement of PUs, they introduced external auctioneer (EA) who is responsible for holding auctions for efficient spectrum allocation.

In [20], auction based approach is used with flexibility of bidding for multiple channels to SUs. It is assumed that full channel side information (CSI) is available and orthogonal frequency division multiplex (OFDM) frame are synchronized. Study for both centralized and distributed control is considered for the uplink of single cell orthogonal frequency division multiplex access (OFDMA) network but the limitation is that ad-hoc networks are not considered where it is difficult to have coordination and synchronization between PUs and SUs for effective cooperative communications.

Macro cell to femto cell switching is another popular technique which attracts mobile operators by promising more coverage and capacity. Concept of femto cell is applicable to many communication standards including wireless code division multiple access (WCDMA), Global system for 
mobile communication (GSM), code division multiple access (CDMA) 2000, Worldwide Interoperability for Microwave Access (WiMax) and long term evolution (LTE) etc. In [21] authors worked on VCG based power control technique for interference minimization between macro cell and multiple femto cell based CRN. A similar technique has been reported in [22] with a difference that authors used multiple macro cell and multiple femto cell based model instead of single macro cell. Coordination between macro cell users and femto cell users is one of the issues which can be considered for future work.

Table1. Comparison of Spectrum allocation techniques using concept, approach, features and limitations.

\begin{tabular}{|c|c|c|c|}
\hline Concept and Approach & System Model & Issues and Future Work & References \\
\hline $\begin{array}{l}\text { Non Cooperative Auction Based } \\
\text { spectrum management technique with } \\
\text { Distributed control. }\end{array}$ & $\begin{array}{l}\text { Single PU network with multiple } \\
\text { SUs. }\end{array}$ & $\begin{array}{l}\text { Application to heterogeneous channel } \\
\text { networks. }\end{array}$ & [9] \\
\hline $\begin{array}{l}\text { Non cooperative game model. } \\
\text { Oligopoly market competition based } \\
\text { modeling of CRN. }\end{array}$ & Single PU with Multiple SUs. & $\begin{array}{l}\text { Demand of Channel choices for SUS. } \\
\text { Further improvement in spectrum } \\
\text { allocation by modeling Multiple PUs } \\
\text { based CRN. }\end{array}$ & {$[10]$} \\
\hline $\begin{array}{l}\text { Static and dynamic Markovian game } \\
\text { model. Use of asynchronous iterative } \\
\text { water filling algorithm to obtain Nash } \\
\text { equilibrium. Distributed power } \\
\text { allocation without coordination. }\end{array}$ & $\begin{array}{l}\text { Multiple CR users (SUs) and multiple } \\
\text { receivers. }\end{array}$ & $\begin{array}{l}\text { Reduction in state space when } \\
\text { number of users increase. } \\
\text { Development of pricing mechanism } \\
\text { other than VCG. }\end{array}$ & {$[11]$} \\
\hline $\begin{array}{l}\text { Cooperative Game model. Spectrum } \\
\text { allocation based on comparison of } \\
\text { channel idle duration and } \\
\text { transmission time required by SUs. }\end{array}$ & Multiple PUs, Multiple SUs & $\begin{array}{l}\text { Perfect channel sensing information } \\
\text { is required for channel allocation. }\end{array}$ & [12] \\
\hline $\begin{array}{l}\text { Non Cooperative VCG game based } \\
\text { model for cognitive radio networks. } \\
\text { Use of Kelly VCG to ensure fair } \\
\text { spectrum utilization. }\end{array}$ & Multiple PUs and SUs. & $\begin{array}{l}\text { Delay minimization for spectrum } \\
\text { allocation, improvement in fairness. }\end{array}$ & [13] \\
\hline $\begin{array}{l}\text { Spectrum management policy based } \\
\text { on VCG mechanism. }\end{array}$ & PS-BS, PS-MS, CR-MSs & Channel gain. & [14] \\
\hline $\begin{array}{l}\text { Modeling Auction mechanism based } \\
\text { on Contract theory. Primary operator } \\
\text { acts a monopolist. Use of algorithm } \\
\text { to find channel quality and price of } \\
\text { spectrum. }\end{array}$ & $\begin{array}{l}\text { PO, Multiple SUs with } \\
\text { Heterogeneous Channel. }\end{array}$ & $\begin{array}{l}\text { SU's may face delay if central } \\
\text { allocator gets overloaded. }\end{array}$ & [15] \\
\hline $\begin{array}{l}\text { Multiple Auctioneer game. Multiple } \\
\text { POs participate as auctioneers. }\end{array}$ & $\begin{array}{l}\text { Multiple POs, multiple PUs and } \\
\text { multiple SUs. }\end{array}$ & $\begin{array}{l}\text { Complexity increases because of } \\
\text { multiple auctioneers. }\end{array}$ & [16] \\
\hline $\begin{array}{l}\text { Auction based spectrum allocation } \\
\text { mechanism for OFDMA based CRN. } \\
\text { SUs provided with flexibility to bid } \\
\text { for multiple channels. }\end{array}$ & PBS, PU, AP and Multiple SUs. & Ad- Hoc networks not considered. & [17] \\
\hline $\begin{array}{l}\text { Non cooperative game model for } \\
\text { spectrum pricing based on duopoly } \\
\text { dynamic spectrum market. Use of } \\
\text { propagation model to reflect } \\
\text { heterogeneity. }\end{array}$ & WSPs and SUs & $\begin{array}{l}\text { Modeling using multiple WSPs and } \\
\text { impact of maximum transmit power } \\
\text { on optimal spectrum price. } \\
\text { Investigation on network } \\
\text { heterogeneity and its impact on } \\
\text { WSP's auction strategy. }\end{array}$ & [18] \\
\hline $\begin{array}{l}\text { Auction model using external } \\
\text { auctioneer concept. EA collects } \\
\text { information from POs and SUs to } \\
\text { hold auction. }\end{array}$ & EA, POs, SUs & $\begin{array}{l}\text { Collusion avoidance, trade off } \\
\text { between efficiency and economic } \\
\text { robustness. }\end{array}$ & [19] \\
\hline $\begin{array}{l}\text { Combinatorial approach for bidding } \\
\text { mechanism. Bidding possible for } \\
\text { multiple frequency bands. }\end{array}$ & $\begin{array}{l}\text { PUs, Spectrum data centers, POs, } \\
\text { SUs. }\end{array}$ & $\begin{array}{l}\text { Submission of multiple bids, online } \\
\text { auction mechanism with time } \\
\text { frequency flexibility. }\end{array}$ & [20] \\
\hline $\begin{array}{l}\text { Resource management for femto } \\
\text { cells. Focus on interference } \\
\text { minimization by using VCG based } \\
\text { power control scheme. }\end{array}$ & Macro cell with Multiple femto cells. & $\begin{array}{l}\text { Delay minimization for spectrum } \\
\text { allocation. }\end{array}$ & {$[21]$} \\
\hline
\end{tabular}


Focus on interference minimization problem between macro cells and femto cells.

\author{
Multiple macro cell and femto cell Coordination between macro cell and \\ users. \\ femto cell users. Improvement in \\ overall network efficiency.
}

\section{CONCLUSION}

Adaptive energy efficient spectrum allocation is a key challenge in self organizing networks. With the rapid development in the field of mobile communication systems, a lot of research work has been done towards realization of flexible and practical CR systems. It includes introduction of new communication techniques such as a) Optical frequency division multiple access (OFDMA) based systems, b) network splitting using small cell or femto cell communication, c) multiple input multiple output (MIMO) systems, and d) addition of more frequency bands to $4 \mathrm{G}$ networks. However, there are still open research issues like a) sensing inaccuracy, b) limited computing capabilities of wireless units, c) coordination between SUs, d) channel switching delay, antenna selection delay in MIMO systems, e) limited power budget of mobile users, and $f$ ) security issues.

From the study presented in this paper, it is clear that practical realization of future envisioned radio systems require optimal energy efficient spectrum management model to facilitate the efficient use of radio spectrum.

\section{REFERENCES}

[1] J. Mitola III and G. Q. Maguire Jr., "Cognitive radio: Making software radios more personal," IEEE Personal Communications, vol. 6, no. 4, pp. 13-18, August 1999.

[2] F. C. Commussion, Spectrum Policy Task Force. Rep. ET Docket no. 02-135, November, 2007.

[3] S. Haykin, "Cognitive radio: Brain-empowered wireless communication," IEEE Journal on Selected Areas in Communication, vol. 23, pp. 201-220, February 2005.

[4] Theodore S. Rappaport, Shu Sun, Rimma Mayzus, Hang Zhao, Yaniv Azar, Kevin Wang, George N. Wong, Jocelyn K. Schulz1, Mathew Samimi1, and Felix Gutierrez1, "Millimeter Wave Mobile Communications for 5G Cellular: It Will Work!", IEEE Access practical innovations: open solution, Vol. 1, 2013.

[5] J. V. Neumann and O. Morgenstern, " Theory of Games and Economic Behavior" Princeton University Press, 1944.

[6] Molisch,A.F., Greenstein,L.J. ; Shafi,M. ," Propagation issues for cognitive radio",Proceedings of the IEEE Volume:97, Issue:5 pp: 787 - 804, 2009.

[7] Xuemin Hong $\dagger$, Cheng-Xiang Wangt, and John Thompson, "Interference Modeling of Cognitive Radio Networks", 2008 IEEE 67th Vehicular Technology Conference-Spring, Marina Bay, Singapore 11-14 May 2008.

[8] Z. Chen et al., "Channel State Prediction in Cognitive Radio, Part II: Single-User Prediction,” Proc. IEEE Southeastcon, pp. 50-54, Mar. 2011.

[9] Xinbing Wang, Zheng Li, Pengchao Xu, Youyun Xu, Xinbo Gao, and Hsiao- Hwa Chen, "Spectrum sharing in cognitive radio networks- An auction based approach" IEEE Transactions On Systems, Man, And Cybernetics Part B

[10] Dusit Niyato and Ekram Hossain, " A Game-Theoretic Approach to
[22] in Cognitive Radio Networks " IEEE Wireless

Communications and Networking Conference, WCNC 2007, Hong Kong, China, 11-15 March, 2007. IEEE 2007 ISBN 1-4244-0658-7

[11] Jane Wei Huang, and Vikram Krishnamurthy, " Game theoretic issues in cognitive radio systems", Journal of communication, Vol. 4, No. 10, November 2009.

[12] Chittabrata Ghosh, Dharma P Agarwal, M Bhaskara Rao, Carlos Cordeiro, "Channel capacity maximization in cooperative cognitive radio networks using game theory", ACM SIGMOBILE Mobile Computing and Communications Review, Volume 13 Issue 2, pp 2-13, April 2009.

[13] Omar El Ferkouss, Wessam Ajib, " Game theory based resource allocation for cognitive radio networks", GLOBECOM 2012: 1174-1179

[14] H.-B. Chang and K.-C. Chen, "Auction-based spectrum management of cognitive radio networks," Vehicular Technology, IEEE Transactions on, vol. 59, no. 4, pp. 1923-1935,May2010.

[15] L. Gao, X. Wang, Y. Xu, and Q. Zhang, "Spectrum trading in cognitive radio networks: A contracttheoretic modeling approach," Selected Areas in Communications, IEEE Journal on, vol. 29, no. 4, pp. 843-855, April 2011.

[16] L. Gao, Y. Xu, and X. Wang, "Map: Multiauctioneer progressive auction for dynamic spectrum access," Mobile Computing, IEEE Transactions on, vol. 10, no. 8, pp. $1144-1161$, Aug. 2011.

[17] Hong $\mathrm{Xu}$, Baochun $\mathrm{Li}$, "Resource allocation with flexible chanel cooperation in cognitive Radio Networkks", IEEE INFOCOM, San Diego, CA, March 2010.

[18] A.W. Min, X. Zhang, J. Choi, and K.G. Shin, "Exploiting Spectrum Heterogeneity in Dynamic Spectrum Market," Mobile Computing, IEEE Transactions on, vol. 11 , no. 12 , pp. $2020-2032$, Dec. 2012.

[19] X. Zhou and H. Zheng, "Trust: A general framework for truthful double spectrum auctions," in INFOCOM 2009, IEEE, pp. 999 - 1007, April 2009.

[20] M. Dong, G. Sun, X. Wang, and Q. Zhang, "Combinatorial auction with time-frequency flexibility in cognitive radio networks," in INFOCOM, 2012 Proceedings IEEE, pp. 2282 -2290, March 2012.

[21] Shao-Yu Lieu, Yu Yu Lin, Kwang-Cheng Chen, " Cognitive and Game Theoretical Radio Resource management for autonomous Femtocells with QoS Guarantees", IEEE transaction on wireless communications, Vol. 10, No. 7, July 2011.

[22] Yongkang Liu, Lin X. CAI, Xuemin (Sherman) Shen, HongWEI Luo, “ Deploying Cognitive cellular networks under dynamic resource management”, IEEE wireless communications, April 2013.8754442 